\title{
Enfermedad cerebrovascular en la infancia: presentación de casos clínicos
}

\author{
Cerebrovascular disease in childhood. Case series
}

\author{
Dra. Ana P. Graziano ${ }^{a}$ Dra. Andrea Sancilio ${ }^{a}$, Dra. Mariela Bugalter ${ }^{a}$ Dr. Walter Barbosa ${ }^{b}$, \\ Dr. Facundo Rodríguezc, Dr. César Montali y Dr. Pablo García Munitis ${ }^{a}$
}

\section{RESUMEN}

La enfermedad cerebrovascular en la infancia es poco frecuente. Su presentación es aguda y constituye una de las 10 causas de mortalidad en pediatría. El objetivo de nuestro estudio es presentar las características de la enfermedad de 18 pacientes menores de 15 años ingresados en el Hospital El Cruce entre julio de 2009 y junio de 2014, y su evolución clínica al año del evento. Observamos un predominio de sexo masculino y una mediana de edad de 5 años. La forma de presentación en orden de frecuencia fue hemiparesia, convulsiones, cefalea y vómitos, y deterioro del sensorio. La forma isquémica fue la más frecuente y el territorio más afectado fue el de la arteria cerebral media. Doce pacientes no presentaron secuelas. Los signos y síntomas fueron orientadores de enfermedad cerebrovascular; los estudios de neuroimágenes, esenciales para su diagnóstico; y los pacientes evolucionaron favorablemente con bajo número de recurrencia y fallecidos.

Palabras clave: accidente cerebrovascular, niños, hemiparesia, convulsiones.

\begin{abstract}
Stroke in childhood is considered rare. It may be ischemic or hemorrhagic. Its presentation is often acute and it is a leading cause of mortality in pediatrics. The aim of our work is to present the general features of stroke of 18 patients under 15 years of age admitted to the Hospital El Cruce, between July 2009 and June 2014, and their clinical outcome a year later. We observed a predominance of male patients and the median age of 5 years. The main clinical features were hemiparesis, seizures, headache and vomiting and sensory impairment. The most frequent type was ischemic and the middle cerebral artery territory was the most commonly involved. Twelve patients had no sequelae. The signs and symptoms were guiding stroke, neuroimaging studies were essential for diagnosis and patients evolved favorably with low number of recurrence and death. Key words: stroke, children, hemiparesis, seizures.
\end{abstract}

a. Cuidados Intermedios Pediátricos.

b. Neurología infantil.

c. Neurocirugía infantil.

Hospital El Cruce de Florencio Varela.

Buenos Aires, Argentina.

Correspondencia:

Dra. Ana P. Graziano: anitagraziano@hotmail.com

Financiamiento: Ninguno.

Conflicto de intereses: Ninguno que declarar.

Recibido: 30-4-2015

Aceptado: 3-8-2015 http:/ /dx.doi.org/10.5546/aap.2016.e5

\section{INTRODUCCIÓN}

La enfermedad cerebrovascular (ECV) es considerada poco frecuente en la edad pediátrica. ${ }^{1-4} \mathrm{La}$ incidencia en niños es de 2,5 a 4,5/100 000 al año. ${ }^{1,2}$ Puede ser de tipo hemorrágico o isquémico y se diferencia de la ECV en adultos por su etiología, historia natural y tratamiento. ${ }^{1}$ A pesar de que la forma de presentación generalmente es aguda, el retraso en el diagnóstico limita las oportunidades para obtener mejores resultados. ${ }^{4,5}$ Las formas de presentación más frecuentemente descritas en la ECV isquémica son la hemiparesia, los signos de foco, las convulsiones y la alteración del estado de conciencia ${ }^{4,6}$ mientras que la ECV hemorrágica se presenta con cefalea, náuseas y vómitos. ${ }^{4,6}$ Las causas de ECV en la infancia son mucho más numerosas y heterogéneas que en adultos, pero su reconocimiento es más dificultoso, con un debut, a veces, insidioso y un curso progresivo, fluctuante o recurrente. ${ }^{2,5}$ Las cardiopatías congénitas representan la principal causa de ECV isquémica y, en un tercio de los casos, no es posible identificar una etiología específica ${ }^{6-8}$ La ECV constituye una de las 10 causas más frecuentes de mortalidad en la infancia. ${ }^{2,3,5,8}$

La supervivencia en niños es mayor que en adultos y las secuelas neurológicas y la mortalidad son altas. ${ }^{4,5}$

El objetivo principal de nuestro trabajo es presentar las características de la ECV en la población infantil hospitalizada en las Unidades de Cuidados Intermedios (CIPED) e Intensivos Pediátricos (UCIP) del Hospital El Cruce y su evolución clínica al año del evento.

\section{CASOS CLÍNICOS}

Se describe una serie de casos clínicos, que incluyó a 18 pacientes de 1 mes a 15 años, que ingresaron a la CIPED y la UCIP del Hospital de Alta Complejidad El Cruce entre julio de 2009 y junio de 2014 con diagnóstico de ECV. 
Definición de ECV según la Organización Mundial de la Salud (OMS): ${ }^{1,5}$ progresión acelerada de signos clínicos, ya sea de alteraciones focales o globales de la función cerebral, con duración de 24 h o más, que puede provocar la muerte con ninguna otra causa aparente más que el origen vascular. Cuando los síntomas se resuelven antes de las 24 horas y sin alteración de la neuroimagen, se denomina accidente isquémico transitorio (AIT). ${ }^{3}$

Se utilizó una historia clínica informatizada como herramienta para el registro, resguardo y recuperación de los datos generados en los procesos asistenciales.

Se analizaron las manifestaciones clínicas al momento de su ingreso y su asociación con el/los territorios vasculares comprometidos en los estudios de neuroimagen, el tipo de $\mathrm{ECV}$, el tiempo de evolución entre la aparición de los síntomas y el diagnóstico de ECV por neuroimagen, la etiología encontrada y el grado de incapacidad según la escala de Rankin al año de su evento.

Los pacientes fueron evaluados por pediatras y profesionales especialistas en neurocirugía y neurología de la Institución. Los pediatras solicitaron tomografía axial computada (TAC) y los especialistas, resonancia nuclear magnética (RNM), angiorresonancia y/o angiografía digital según el caso.

El seguimiento fue realizado por un neurólogo y un neurocirujano infantil, y un pediatra por consultorio externo con evaluación clínica y estudios por imágenes según el caso. Se realizó cada 2-6 meses de acuerdo con el estado clínico del niño y la causa. Para evaluar la incapacidad al año del evento, se utilizó la escala de Rankin modificada. ${ }^{9,10}$ Fue originalmente introducida en 1957 por Rankin y modificada a su forma aceptada actualmente a finales de 1980 (Tabla 1). Es una herramienta útil para intentar cuantificar de manera fiable y precisa la gravedad de la enfermedad y su progresión. Trata de medir lo que los pacientes son capaces de realizar en la vida diaria para compararlo con lo que podían o podrán hacer intentando medir autonomía personal.

Ingresaron 18 pacientes con diagnóstico de ECV; 14/18 fueron de sexo masculino; la mediana de edad fue de 5 años, con un rango entre 1 mes y 15 años. El tiempo de evolución transcurrido desde el inicio de los síntomas hasta el momento del diagnóstico por imagen de ECV fue de $12 \mathrm{~h}$ en $4 / 18$, de 24 h en $8 / 18$ y de 48 h en $6 / 18$ pacientes. El tipo de ECV más frecuentemente observado fue el isquémico, en 11/18. La forma de presentación más habitual de ECV fue hemiparesia en $8 / 18$ de los casos, seguida de convulsiones en $6 / 18$, cefalea más vómitos en $4 / 18$ y deterioro del sensorio en $3 / 18$. El territorio vascular comprometido con mayor frecuencia correspondió al de la arteria cerebral media, en 10/18. En la Tabla 2, se puede observar la asociación entre signos y síntomas con el territorio vascular y cerebral comprometido.

Según el mecanismo fisiopatogénico de la enfermedad, isquémico o hemorrágico, hubo un predominio de síntomas de foco en el primero e hipertensión endocraneana y alteración del sensorio en el segundo.

Las causas de la ECV de origen isquémico fueron desconocidas en $3 / 18$, por cardiopatía congénita en $3 / 18$, secundarias a miocardiopatía adquirida en 2/18, infecciosas en 2/18 ( 1 caso de varicela y 1 caso de coqueluche) y por trombofilia en $1 / 18$.

Las causas de la ECV de origen hemorrágico fueron secundarias a malformación arteriovenosa (MAV) en 3/18, por alteraciones de la hemostasia

TABLA 1. Escala de Rankin modificada

\section{Sin síntomas}

1. Sin incapacidad importante

2. Incapacidad leve

3. Incapacidad moderada

4. Incapacidad moderadamente grave

5. Incapacidad grave

6. Muerte
Capaz de realizar sus actividades y obligaciones habituales.

Incapaz de realizar algunas de sus actividades previas, pero capaz de velar por sus intereses y asuntos sin ayuda.

Síntomas que restringen de modo significativo su estilo de vida o impiden su subsistencia totalmente autónoma (p. ej., necesita alguna ayuda).

Síntomas que impiden claramente su subsistencia independiente, aunque sin necesidad de atención continua (p. ej., incapaz para atender sus necesidades personales sin asistencia).

Totalmente dependiente; necesita asistencia constante día y noche. 
en 3/18 y por hipertensión arterial en 1/18.

Los estudios de neuroimagen fueron solicitados de acuerdo con la necesidad asistencial de cada caso particular, debido a no contar con guías de diagnóstico. Se realizó TAC a todos los pacientes. El resto de los estudios realizados fueron RNM a 10/18, angiografía digital a 9/18 y angiorresonancia a $7 / 18$.

Luego de un año de seguimiento y según la evaluación a través de la escala de Rankin, 12/18 pacientes no presentaron secuelas (Rankin 0), $1 / 18$ presentó puntuación $1,1 / 18$ correspondió a la escala de Rankin 3 y 2/18 fallecieron (Rankin 6). Dos pacientes no continuaron el seguimiento.

Al evaluar la evolución según la escala de Rankin y el tiempo al momento del diagnóstico, se observó que, entre los 12 pacientes que no presentaron secuelas, 2 se diagnosticaron dentro de las 12 horas, 6 dentro de las 24 horas y 4 en 48 horas.

De los 18 pacientes observados, 2 evolucionaron a la muerte. Uno de estos pacientes falleció durante el evento inicial de ECV y por dicha causa; el segundo paciente falleció por recurrencia del sangrado, cuya causa era MAV.

\section{DISCUSIÓN}

La enfermedad cerebrovascular en pediatría es considerada rara y poco frecuente, lo que contribuiría a un retraso diagnóstico. ${ }^{4}$ Actualmente, se observa un aumento en el diagnóstico de la ECV debido al mayor acceso a estudios de neuroimágenes y al mayor conocimiento de factores de riesgo asociados a ECV. ${ }^{8}$ En nuestro estudio, el tipo de ECV (isquémica), sus causas, la mediana de edad de 5 años y el predominio del sexo masculino coinciden con la mayoría de la bibliografía publicada. ${ }^{1,2,5,8,11,12}$

La forma de presentación más frecuente en la ECV de origen isquémico fue la hemiparesia y las convulsiones, y en la ECV de origen hemorrágico, correspondió a síndrome de hipertensión endocraneana y compromiso del nivel de conciencia, lo que coincidió con las publicaciones de otros autores. ${ }^{1,3,4,7,11}$

El diagnóstico de esta patología se realiza principalmente por neuroimagen. La TAC es el método inicial, pero puede no detectar lesiones en el período agudo, por lo que la RNM y la angiorresonancia son las técnicas de elección. ${ }^{3,4,6,8}$ Se deben solicitar estudios de laboratorio según la sospecha diagnóstica para detectar estados protrombóticos, causas hematológicas, trastornos metabólicos, enfermedades autoinmunes e infecciosas que causen vasculitis, buscar cardiopatías congénitas y alteraciones vasculares (disección arterial)., ${ }^{1,2,6}$

En cuanto al pronóstico, se ha referido que las lesiones subcorticales tienen mejor pronóstico que las corticales. ${ }^{1}$ Se han descrito tratamientos anticoagulantes y antitrombóticos para el episodio agudo en niños, extrapolados de adultos, pero aún faltan estudios que indiquen que sean seguros y eficaces en pedíatra. El ácido acetil salicílico (AAS) es relativamente seguro a bajas dosis. Se podría indicar heparina de bajo peso molecular en niños con ECV cardioembólica, por disección arterial e hipercoagulabilidad persistente. ${ }^{6}$ En nuestro trabajo, el tratamiento utilizado fue quirúrgico en los casos de MAV y AAS en las causas cardiológicas.

Son escasos, a la fecha, los estudios que permiten establecer guías de manejo diagnóstico basadas en la evidencia, lo cual hace que la solicitud de estudios de neuroimagen se base en experiencias individuales o extrapolación de recomendaciones para adultos. ${ }^{13}$ Este inconveniente se vio reflejado en los pacientes de nuestro estudio, en el que se encontraron diferencias en cuanto al tipo de estudios solicitados. ${ }^{2,4,6,8,14}$ Debido al escaso número de pacientes, no se pudo establecer una relación entre el tiempo al momento del diagnóstico y el valor

TABLA 2. Asociación entre signos y sintomas y territorio afectado

Síntomas/signos $\left(\mathrm{n}^{\circ}\right.$ de pacientes)

Hemiparesia, afasia ${ }^{10}$

Convulsiones ${ }^{6}$

Compromiso del nivel de conciencia ${ }^{5}$

Hipertensión endocraneana ${ }^{3}$

Síndrome meníngeo ${ }^{1}$
Territorio comprometido $\left(\mathrm{n}^{\circ}\right.$ de pacientes)

$$
\text { Isquemia cerebral media }{ }^{7}
$$

Isquemia cerebral media ${ }^{4}$, hemorragia subaracnoidea y subdural ${ }^{1}$, isquemia cerebral posterior ${ }^{1}$

Isquemia cerebral media ${ }^{2}$, hemorragia intraventricular ${ }^{2}$, hemorragia de ganglios basales ${ }^{1}$

Hemorragia cerebelosa $^{1}$, intraparequimatosa ${ }^{1}$ e intraventricular ${ }^{2}$

Hemorragia intraparenquimatosa 
de la escala de Rankin al año de seguimiento. La mayoría de los pacientes de nuestro estudio no presentaron secuelas. Las recurrencias, al igual que la mortalidad, resultaron menores a las publicadas por otros autores, $3,6,8,11$ pero se debe tener en cuenta que el número de pacientes del estudio es pequeño.

El diagnóstico inicial de ECV en nuestro estudio fue rápido y los signos y síntomas de presentación, orientadores. Resulta imprescindible la confección de guías diagnósticas para los estudios de neuroimagen. La oportunidad de la derivación, la disponibilidad de neuroimagen y de complejidad acorde, tanto para el diagnóstico como para el tratamiento y seguimiento, resultarán determinantes para su abordaje. Estas cuestiones exceden el rol del pediatra asistencial, y el trabajo de gestión y coordinación entre niveles asistenciales juega un papel fundamental para el manejo integral de estos pacientes.

\section{REFERENCIAS}

1. Nunes T, Recalde L, Espinola de Canata M. Características delos accidentes cerebrovasculares (ACV) en niños. Pediatr (Asunción) 2008;35(1):18-23.

2. Ruiz del Olmo-Izuzquiza I, De Arriba-Muñoz A, LopezPison J, Garcia-Iñiguez JP, et al. Accidente cerebrovascular en pediatría. Nuestra experiencia de 18 años. Rev Neurol 2010;50(1):3-11.

3. De Castro de Castro P, Vázquez López M. Accidentes cerebrovasculares en el niño y en el adolescente. En: Asociación Española de Pediatría, Sociedad Española de Neurología Pediátrica, eds. Protocolos diagnósticos y terapéuticos en pediatría: Neurología. 2. ${ }^{a}$ ed. Madrid: AEP; 2008:262-70. [Acceso: 20 de julio de 2015]. Disponibleen:http:/ /www.aeped.es/sites/default/files/ documentos/35-acv.pdf.

4. Cárdenas JF, Rho JM, Kirton A. Pediatric stroke. Childs Nerv Syst 2011;27(9):1375-90.

5. Hernández M, Samsó C, Escobar R, Mesa T. Accidente vascular encefálico. Presentación clínica y factores de riesgo. Rev Chil Pediatr 2013;84(4):396-402.

6. Caycedo L, Piñeros M. Guías de tratamiento en enfermedad cerebro vascular en niños. [Acceso: 20 de julio de 2015]. Disponibleen:http://www.acnweb.org/guia/g6cap23.pdf.

7. Lyle CA, Bernard TJ, Goldenberg NA. Childhood arterial ischemic stroke: a review of etiologies, antithrombotic treatments, prognostic factors, and priorities for future research. Semin Thromb Hemost 2011;37(7):786-93.

8. Lynch JK, Hirtz DG, DeVeber G, Nelson KB. Report of the National Institute of Neurological Disorders and Stroke workshop on perinatal and childhood stroke. Pediatrics 2002;109(1):116-23.

9. Frankel MR, Morgenstern LB, Kwiatkowski T, Lu M, et al. Predicting prognosis after stroke: a placebo group analysis from the National Institute of Neurological Disorders and Stroke rt-PA Stroke Trial. Neurology 2000;55(7):952-9.

10. Rankin J. Cerebral vascular accidents in patients over the age of 60. II. Prognosis. Scott Med J 1957;2(5):200-15.

11. Arroyo HA, Tamer I. Enfermedad cerebrovascular en la infancia y la adolescencia. Accidente isquémico cerebral. Rev Neurol 2002;34(2):133-44.

12. Huici-Sánchez M, Escuredo-Argullós L, Trenchs-Sáinz de la Maza V, Luaces-Cubells C. Enfermedad cerebrovascular en pediatría. Experiencia de un servicio de urgencias. Rev Neurol 2014;59(3):106-10.

13. Engelman KA, Jordan LC. Outcome measures used in pediatric stroke studies: a systematic review. Arch Neurol 2012;69(1):23-7.

14. Michelson AD. Arterial ischemic stroke in children: baby steps. Circulation 2006;114(20):2094-5. 